\title{
Increase the Efficiency of IoT Devices by Using the Wireless Power Transmission in the Industrial Revolution 4.0
}

\author{
https://doi.org/10.3991/ijoe.v17i07.24143 \\ Mohamed Zied Chaari ${ }^{(凶)}$, Somaya Al-Maadeed \\ Qatar University, Doha, Qatar \\ chaari_ziedeieee.org
}

\begin{abstract}
Today's world is evolving toward creating a smart house where a multitude of Internet of Things (IoT) devices and sensors are interacting to deliver plenty of useful information. Essential to the implementation of this IoT is the design of energy-efficient solutions aiming toward a low-carbon-emission, namely green, society. Many R\&D studies are working on a long-range distance wireless charging that will send microwave energy to powered IoT devices wherever it is in the room and without cords. Wireless power transmission technology is the diffusion of RF power without using any physical support. The vision of future technology is to make the IoT devices charging and powered wirelessly. The objective of the work scope is to combine the wireless power technology with smart IoT and IIoT devices and reduce the turnoff of devices due to battery depletion. In this research paper, we designed and realized a wireless charging device using the fundamentals of microwave radiation. The proposed electronic device takes all parameters above to deliver sufficient energy to charging any IoT devices wirelessly with a maximum power of $5 \mathrm{~W}$.
\end{abstract}

Keywords-RF harvesting energy, Wireless Power Transmission, loop antenna, Internet of Things, RF-to-DC converter, Industrial Internet of Things

\section{Introduction}

Nowadays, it is commonplace for cities to be equipped with free WiFi hotspots, real-time traffic information, and safety surveillance, to mention a few. In this respect, creativity-driven and farsighted governments are playing a crucial role in speeding up the city's technological evolution [1-3]. From technological innovations, products and services will become ubiquitous in future smart cities depending on the IoT technology [4] [5]. IoT technology thereby revolutionizinga broad range of applications in a variety of domains, such as healthcare, homeautomation, transportation, intelligent energy management, and smart grids [6]. With ever-increasing services and cloud connectivity, IoT devices set to permeate all aspects of our daily lives [7]. Nevertheless, electricity distribution to power it still relies on cables for its delivery. To increase the performance of the IoT devices, we will implement a combination between the Wireless Power Transmission (WPT) and the Internet of things (IoT). The wireless power tech- 
nology will become a cornerstone in the design of new and grown human settlements in industry 4.0. In this paper, we propose the wireless power technology to charge any IoT devices in the smart district, as shown in Figure 1.
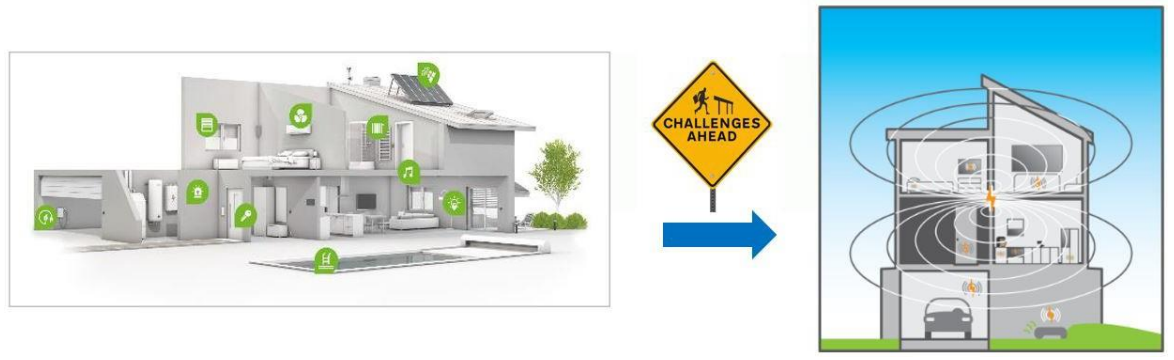

Fig. 1. 3D picture of a smart house without wire

The RF energy generated by a local Microwave Power Station (MPS) [8-10]. Although, still inan early stage, long-range wireless power transmission is gaining momentum.Both industrials and academic know us well that the WPT will be the solution of battery autonomy and running off [11-15].

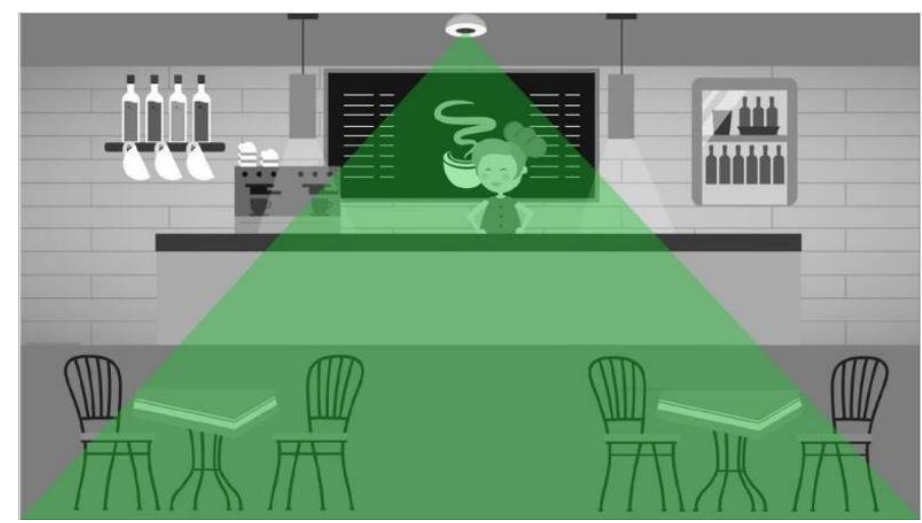

Fig. 2. IoT devices powered and charging wirelessly

This research is toward creating smart IoT devices powered wirelessly to reduce the running out of the battery of the devices, as shown in figure 2. In this study, the portable microwave station already exists and will use in the testing phases.

\section{Design of the Circular Loop Harvester Antenna}

Wireless power harvester devices emphasize lightweight, compact, and low cost to be merged into IoT devices. The efficiency of this antenna depends upon their geometry shape and physical dimensions [16, 17]. Circular loop antenna rises like the right receiver meeting these requirements. This is due to its versatility of possible geometry 
and pure integrity with any IoT and IIoT devices, as shown in figure 3. In this research work, the size of the antenna is a significant parameter for harvesting more energy from different angles and directions. Typically, useful over a range of frequencies relative to the diameter of the wavelength, especially it's hidden inside IoT devices $[18,19]$. The ratio of the radiation intensity is related to the beamwidth of the loop antenna. Where:

The operating frequency $f_{r}=2.45 \mathrm{GHz}$

Speed of light $C=3 \times 10^{8} \mathrm{~m} / \mathrm{s}$

\subsection{Dimension of the receiver antenna}

This antenna works in the UHF range (up to $3 \mathrm{GHz}$ ). The currents throughthis circular antenna will be in the phase. The EM field will be perpendicular to the whole loop carrying the current. The radius is (a) and is assumed to be much smaller than a wavelength $(a<\lambda)$. A small loop is by definition a loop of constant current. Its radius must satisfy:

$$
a<\frac{\lambda}{6 \times \pi} \approx 0.053 \lambda
$$

In fact, to make sure that the current has near-constant distribution along the loop, a tighter limit must be imposed:

$$
a \cdot<\cdot 0.03 \lambda
$$

Here, we give only the far-field components of the loop, the axis of which isalong Z:

$$
\begin{aligned}
\underline{E_{\theta}} & =\left(\eta \beta^{2}\right) \times(I A) \frac{\rho^{-j \beta r}}{\Delta \pi r} \times \sin \theta \\
\underline{H_{\phi}} & =\left(-\beta^{2}\right) \times(I A) \frac{\rho^{-j \beta r}}{\Delta \pi r} \times \sin \theta
\end{aligned}
$$

It is obvious that the far-field pattern:

$$
\overline{E_{\theta}}(\theta) \cdot=\cdot \sin \cdot \theta
$$

Radiated power:

$$
11=\frac{1}{12 \pi}\left(\eta \beta^{4}\right)(I A)^{2}
$$


Radiation resistance:

$$
R_{r}=\eta_{3}^{8}(\pi)^{3}\left(\lambda_{2}\right)^{2} \approx 31171\left(\lambda_{2}\right)^{2}
$$

Equation (7) gives the radiation resistance with one circular loop.

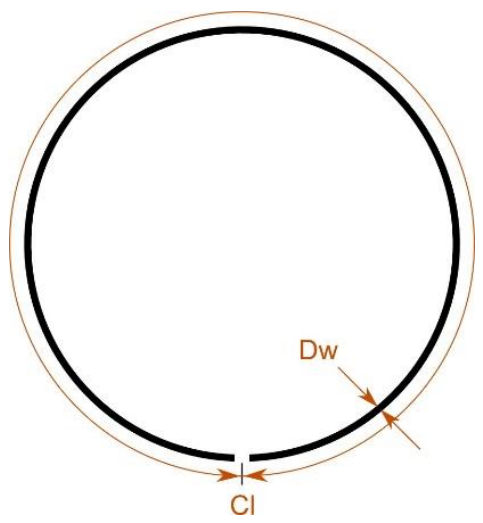

Fig. 3. The geometry of the proposed circular loop antenna

Tabulated values using the above equations shown in Table 1.

Table 1. Overview of the antenna specifications

\begin{tabular}{|l|l|}
\hline \multicolumn{1}{|c|}{ Description } & \\
\hline Operating frequency & $2.45 \mathrm{GHz}$ \\
\hline Wire diameter (DW) & $1 \mathrm{~mm}$ \\
\hline Copper conductivity & $5.8 \mathrm{e} 7 \mathrm{~S} / \mathrm{m}$ \\
\hline Circumference Loop (Cl) & $137 \mathrm{~mm}$ \\
\hline Radius (a) & $21.80 \mathrm{~mm}$ \\
\hline Antenna impedance (Rin) & $50 \Omega$ \\
\hline
\end{tabular}

The optimized parameters of the designed antenna shown in Table 1 and theperformance results will be studied and analyzed in the following subsection.

\subsection{Simulation and discussion}

The layout of the antenna created using the ADS Keysight software, and itis further validated by confirming these results utilizing Microwave Studio soft- ware (CST). We affected many parameters like: Directivity, Reflection coefficient, Smith plot, Radiation pattern 3D, and the Current distribution.

Directivity: The simulated Gain for the designed antenna was $4.78 \mathrm{dBi}$ at 2.45 $\mathrm{GHz}$, and the Gain was $4.97 \mathrm{dBi}$ at $2.25 \mathrm{GHz}$, as shown in figure 4 . 


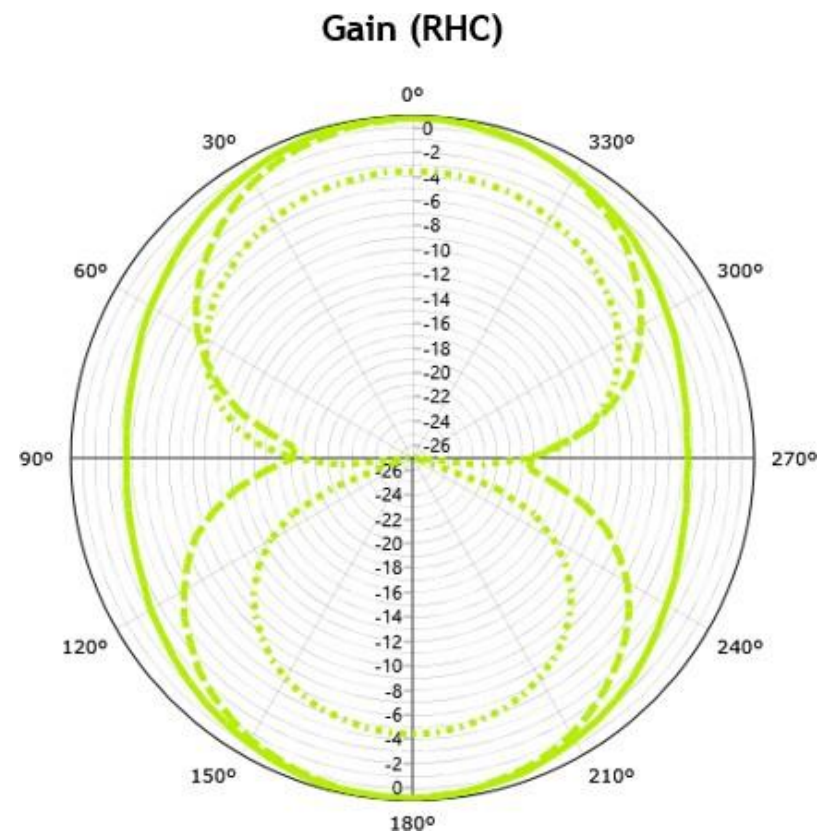

Fig. 4. Directivity of the proposed antenna

Reflection coefficient of the antenna: Return loss $\left(S_{11}\right)$ presented in Figure 5, and we obtain a very high return loss, $-31.21 \mathrm{~dB}$ at the operating frequency. The bandwidth of the receiver antenna is $16.93 \%$.

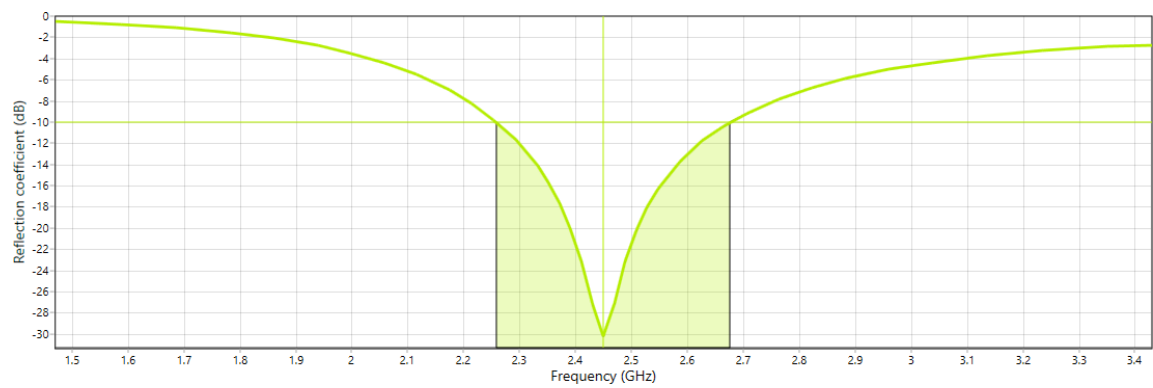

Fig. 5. Antenna Return Loss vs. Frequency

VSWR: A VSWR approaching 1.0 is desired for the loop antenna design at the resonant frequency. At $2.45 \mathrm{GHz}$, the simulation yielded a VSWR around 1.17, as shown in Figure 6.

Radiation pattern 3D: Figure 7 shows the 3D radiation pattern of the pro- posed antenna, which indicates that there are proper lobes and a maximum Gain of $-3 \mathrm{~dB}$. 


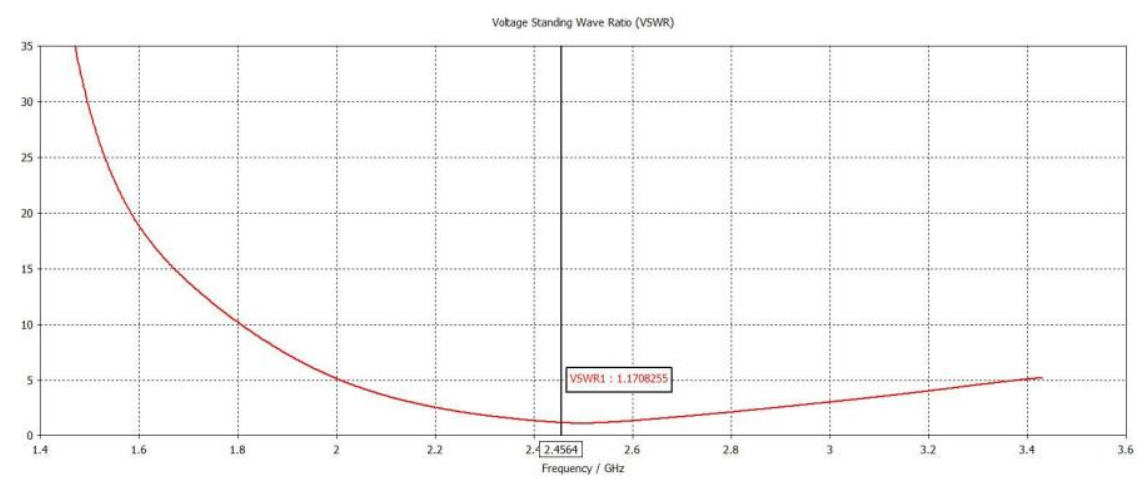

Fig. 6. Antenna VSWR vs. Frequency

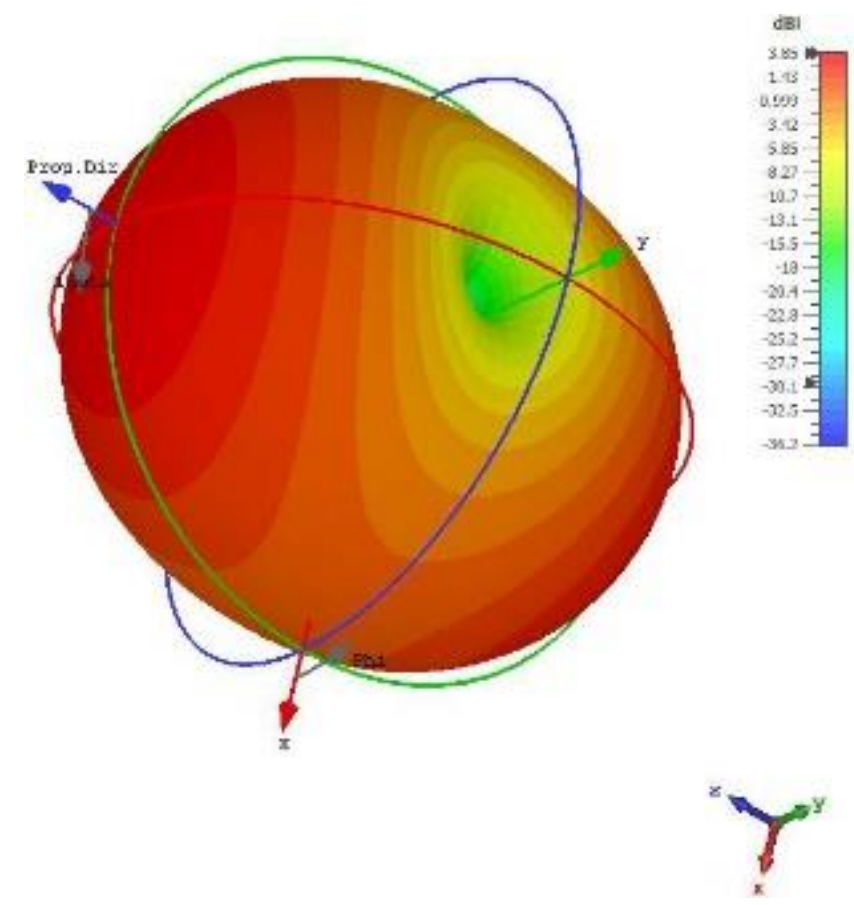

Fig. 7. Radiation pattern of the presented antenna for $2.45 \mathrm{GHz}$,

E-field distribution: Figure 8 shows the simulated E-field distribution of the antenna for different frequencies, as well as $2.45 \mathrm{GHz}$. The E-field intensity $185(\mathrm{~V} / \mathrm{m})$. 

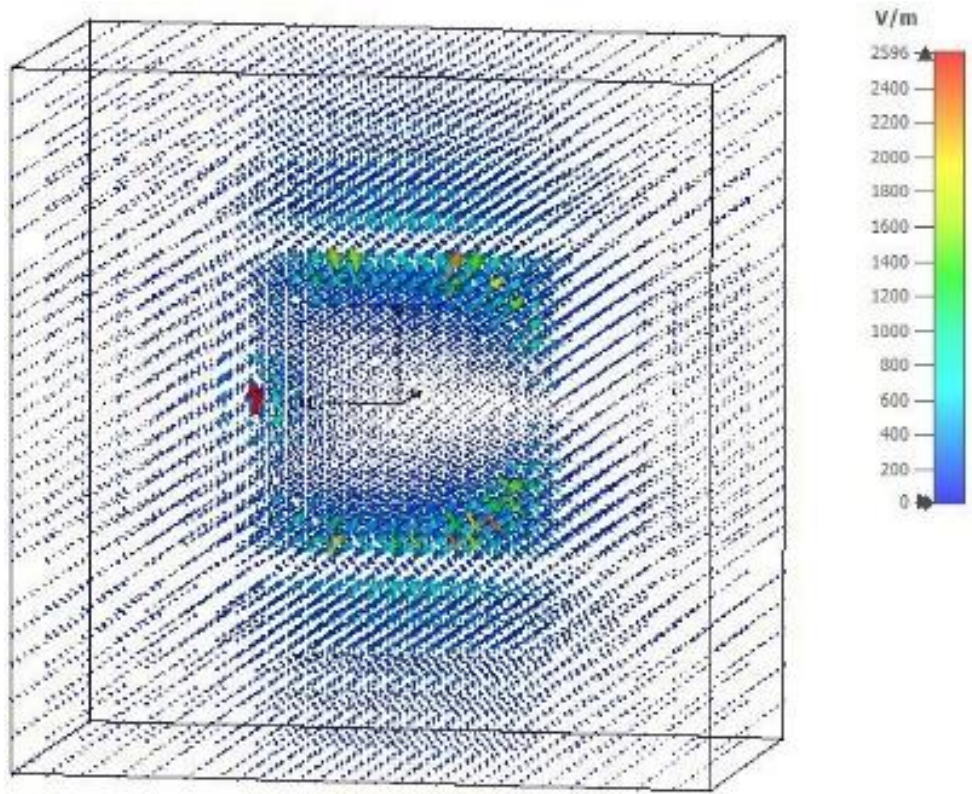

Fig. 8. E-field intensity of the proposed antenna

Current distribution: The 3D current distribution plot gives the relationship between the co-polarization (desired) and cross-polarization (undesired) components. Figure 9 shows the current distribution of the loop antenna.

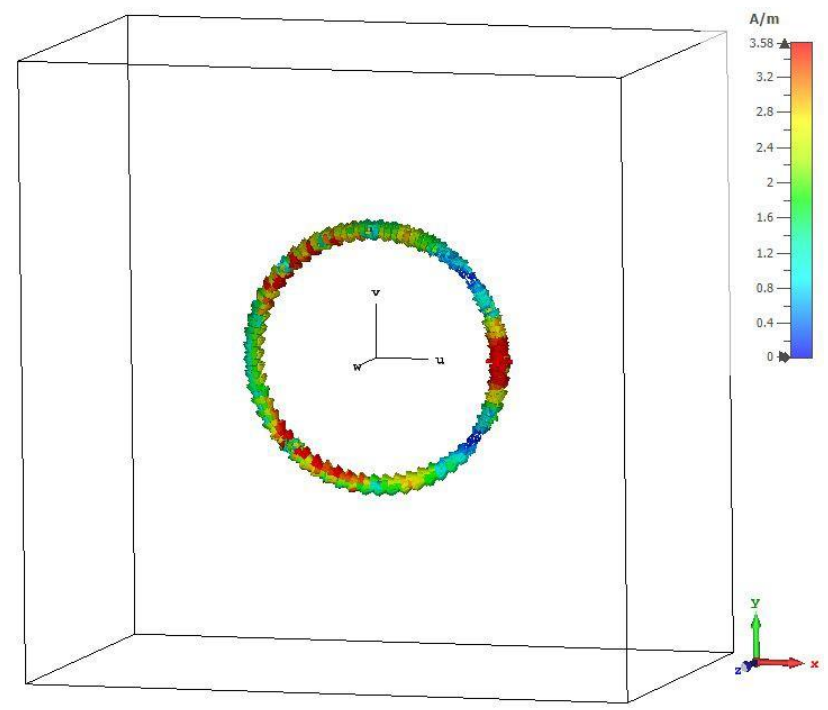

Fig. 9. Surface current distribution of the antenna 
Precisely, the designed antenna has a simulated gain of $4.78 \mathrm{dBi}$, the directivity of $3.849 \mathrm{dBi}$, the input power of $495 \mathrm{~mW}$, and the radiated power of $565 \mathrm{~mW}$ at $f_{r}$ $=2.45 \mathrm{GHz}$.

\section{Energy Harvester Design at $2.45 \mathrm{GHz}$}

This microwave signal is transferred to the 7-stage charge pump rectifier through an impedance matching circuit, to convert into DC power, which is additional accumulated in a storage element. The rectifier is a Schottky diode in which impedance is matched to the antenna by a low pass filter [20-22]. The topologydesign contains three main subsystems, each having specific purposes. The receiver antenna captures electromagnetic radiation from the MPS. The receiversignal is then efficient via the rectifier circuit that utilizes BAT63 (Infineon) Schottky diodes. A final subsystem acts as a backup system by storing powerin the battery. The first storage device stores and accumulates charge from the rectified DC signal outputted by the rectifier circuit. As for the rectifier topologies, the choice of the rectifier, it will be studied and analyzed in the following subsection. All the requirements to install a harvester device hidden into the IoT devices should be proved, as shown in Figure 10.

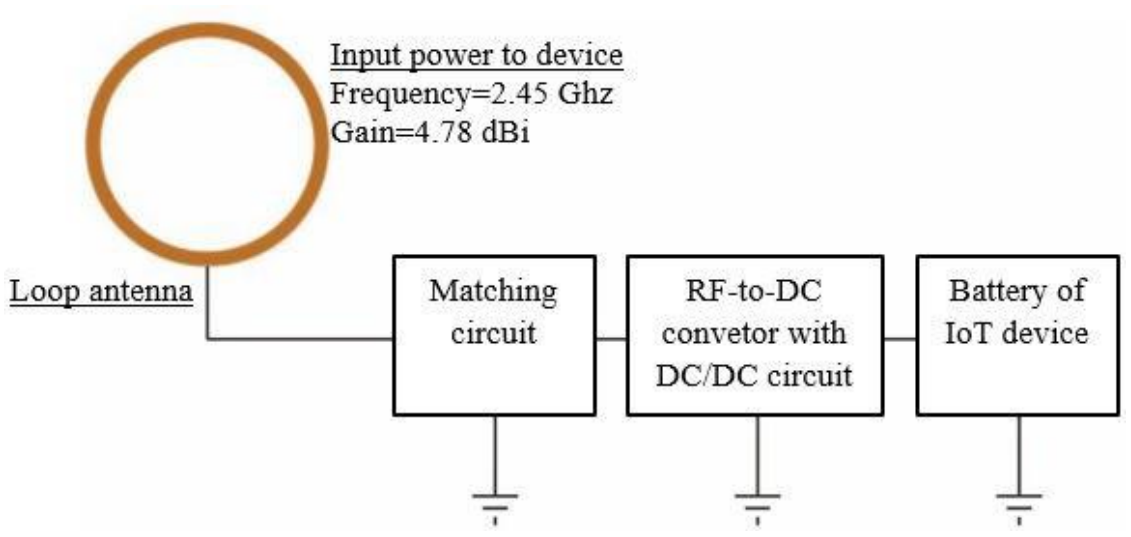

Fig. 10. Block diagram of the proposed harvester circuit

\subsection{Matching circuit}

In the phase testing, the VSWR at $2.45 \mathrm{GHz}$ is 1.17 to reduce to 1 . The matching circuit ensures the maximum power distribution from the antenna to the rectifier circuit [23].

\subsection{Rectification subsystem}

In this part, the RF-DC conversion circuit using seven stages charge pump uitilize Schottky diodes. The input impedance of the rectifier is connected to thematching cir- 
cuit's output to maximize the power harvesting and decrease the signal reflected from the load [24]. Knowing the receiving is designed to operate at $2.45 \mathrm{GHz}$ with the output impedance of $50 \Omega$. Figure 11 shows the schematic de-sign of the rectification subsystem circuit. Figure 12 shows the simulation result of the 7-stage charge pump rectifier using diode BAT63. The below simulation shows the output voltage (V) of the rectifier circuit.

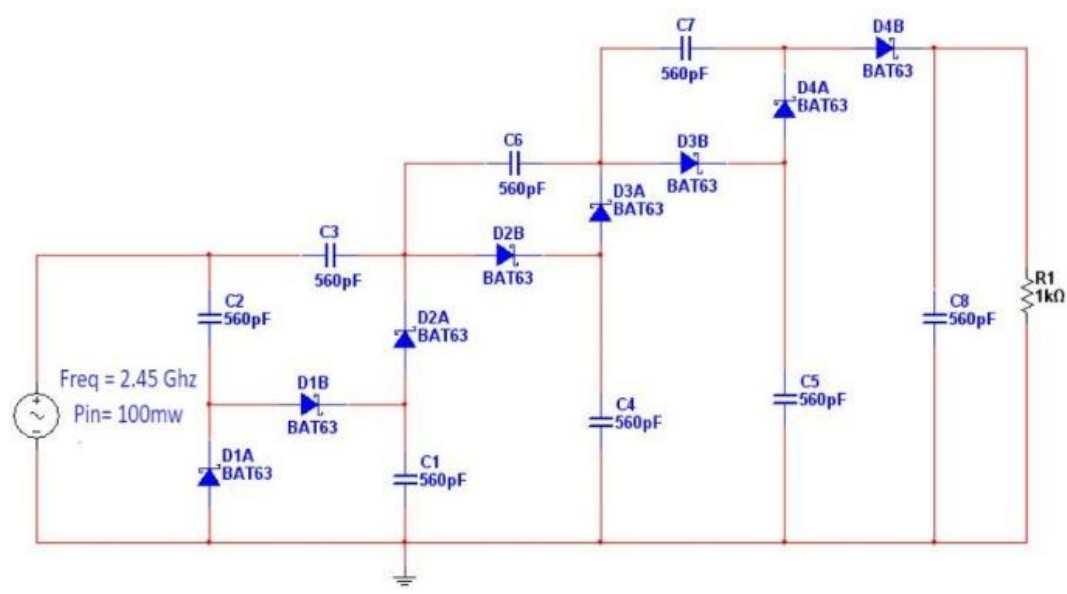

Fig. 11. Schematic diagram of microwave harvesting circuit with BAT63 diode (AgilentADS and Multisim)

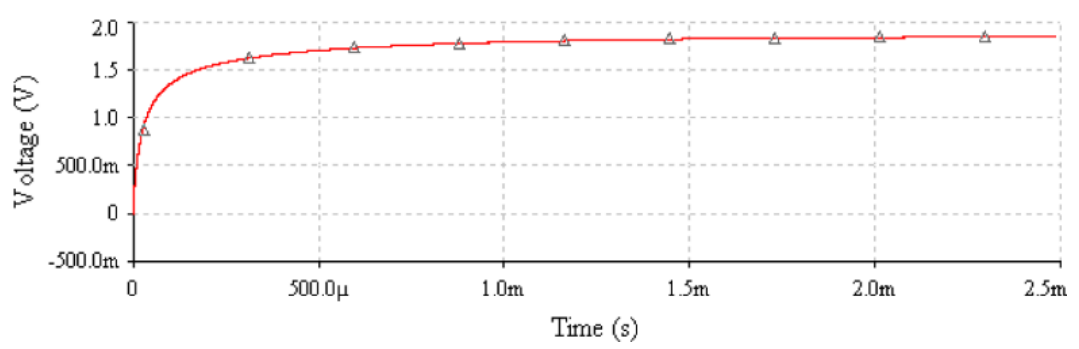

Fig. 12. Transient Analysis for $2540 \mathrm{MHz}$ Charge Pump Design (100mW)

\section{$4 \quad$ Prototype Result and Discussion}

The microwave output power of the microwave station is $P_{\text {out }}=55 \mathrm{~W}$. We create the antenna using high-quality copper. The antenna prototypes are successfully built and measured using the S3101 Antenna Analyzer. Then, the loop antenna is ready to be tested with a vector network analyzer machine VNA. The measuredS11 graph is depicted in Figure 13 in the Magnitude plot. It was around $-24.65 \mathrm{~dB}$ from $2.455 \mathrm{GHz}$, and it is not the same simulation results. Also, our loop antenna can reach a maximum gain of $4.78 \mathrm{dBi}$. 


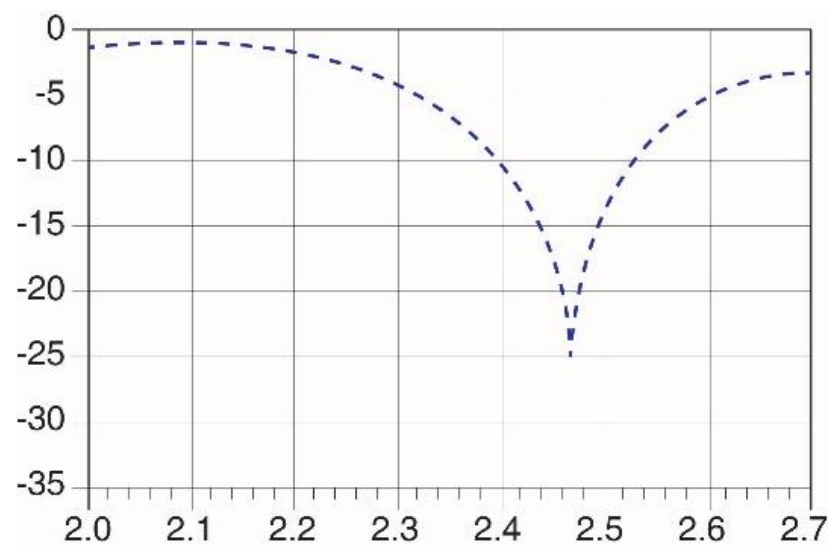

Fig. 13. Measured return loss (S11) results of antenna

The radiation efficiency is $47.23 \%$. All the antenna parameters have been tested and optimized to deliver high-performance receivers. The results are satisfactory and show the advantage of using a loop antenna for acquiring microwave energy. In summary, all the simulated and real measurements indicate that theharvester device has a good performance. The energy acquiring device of the present work is capable of receiving microwave power from the S-band, and con- vert the received signals into electrical energy. An examination of the prototypein Figure 14 and Figure 15, respectively.

Actual test, it can be observed the efficiency of the harvester circuit changesaccording to the distance. The amount of power received change according to the gap between the microwave power station and the harvester circuit, as shown intable 2.

\section{Conclusion and Perspectives}

In this research paper, we tested, and we approved that we could produce electricity from S-band frequency to charge any battery of electronic devices. We made sure to easily install the harvester circuit into any IoT devices. The antenna achieves a reflection coefficient in a real test of $-21.351 \mathrm{~dB}$ and the maximum gain direction at $4.80 \mathrm{~dB}$ at the operating frequency. All these performances measured results make this antenna suitable for S-band. High power and high-efficiency RF-to- DC convertor fabricated using Schottky diodes BAT63. 


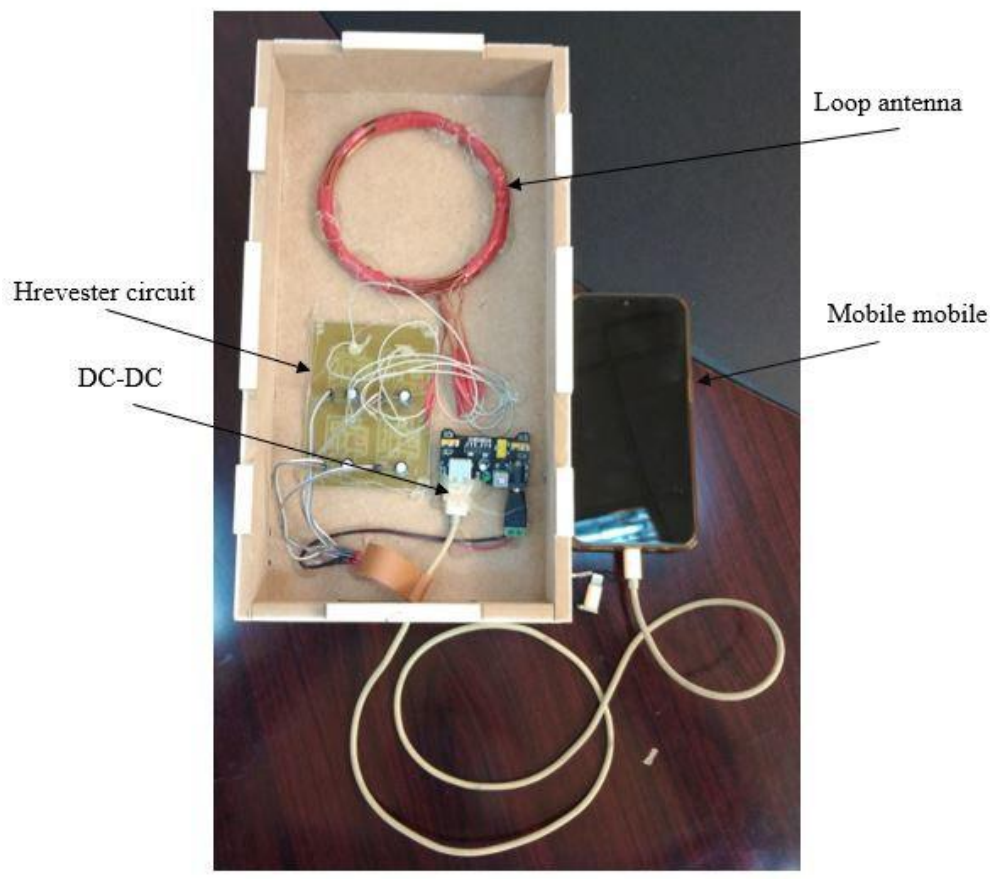

Fig. 14. Photograph of the receiver prototype (Photograph of the microwave harvesterdevice connected to a mobile phone to charge it)

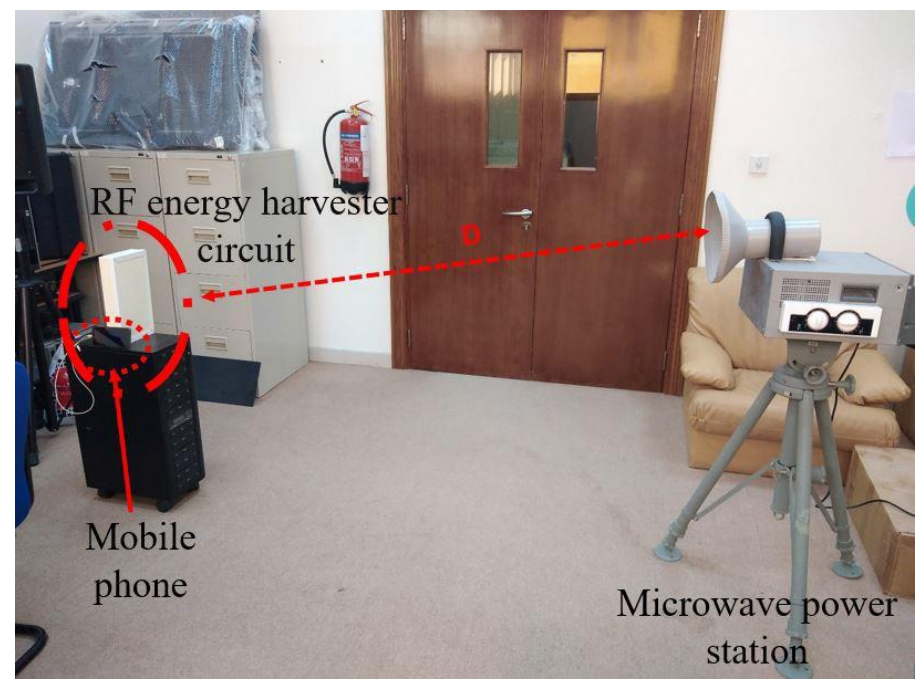

Fig. 15. Photograph of the experimental setup with a prototype

The circuit achieves high output DC voltage and good conversion back into electricity efficiency. The conversion efficiency of $27 \%$ was achieved at S-band at an input 
power level of $14.77 \mathrm{~W}$ a distance $10 \mathrm{~m}$. In this research, we approved that we could change the strategy of the future industry 4.0 using the wireless power technology to keepall IoT devices full charge and can working a maximum of the time. In the future should be all IoT and IIoT electronic devices powered wirelessly. For the next work, different types of miniature antenna can be used in this application. We will also change the topology of the rectiier circuit and check the efficiency of the RF-to-DC circuit.

Table 2. The efficiency of the harvest circuit depends on the gap distance between the RF emitter and receiver IoT device

\begin{tabular}{|c|c|}
\hline Distance between emitter and the Harvesting circuit $(\mathbf{m})$ & Power at the receiving point $(\mathbf{W})$ \\
\hline 10 & 14.77 \\
\hline 15 & 13.58 \\
\hline 20 & 10.23 \\
\hline 25 & 8.01 \\
\hline 50 & 6.55 \\
\hline
\end{tabular}

\section{References}

[1] Longoria, G. et al.: Wireless Power Transmission in Smart Cities: The wIs- hood - Wireless Smart Neighborhood: In: Proceedings of the 6th International Conference on Smart Cities and Green ICT Systems. pp. 317322 SCITEPRESS - Science and Technology Publications, Porto, Portugal (2017). https://doi.org/10.5220/0006364003170322

[2] Shu, J. et al.: Molecular Patching Engineering to Drive Energy Conversion as Efficient and Environment Friendly Cell toward Wireless Power Transmission. Adv. Funct. Mater. 30, 10, 1908299 (2020). https://doi.org/10.1002/adfm.201908299

[3] Smartgreens: Smartgreens 2017: Proceedings of the 6th International Conference on Smart Cities and Green ICT Systems: Porto, Portugal, April 22- 24, 2017. SCITEPRESS - Science and Technology Publications, Lda, Setbal (2017). https://doi.org/10.1007/978-3-030$\underline{02907-4}$

[4] Li, L. et al.: A Prediction-Based Charging Policy and Interference Mitigation Approach in the Wireless Powered Internet of Things. IEEE J. Sel. Areas Commun. 37, 2, 439451 (2019). https://doi.org/10.1109/JSAC.2018.2872429

[5] Lin, W., Ziolkowski, R.W.: A Circularly Polarized Wireless Power Transfer System for Internet-of-Things (IoT) Applications. In: 2020 4th Australian Microwave Symposium (AMS). pp. 12 IEEE, Sydney, Australia (2020). https://doi.org/10.1109/ams48904.2020. $\underline{9059529}$

[6] Farmanbar, M. et al.: A Widespread Review of Smart Grids Towards Smart Cities. Energies. 12, 23, 4484 (2019). https://doi.org/10.3390/en12234484

[7] Suryadevara, N.K., Biswal, G.R.: Smart Plugs: Paradigms and Applications in the Smart City-and-Smart Grid. Energies. 12, 10, 1957 (2019). https://doi.org/10.3390/en12101957

[8] Zhu H, Xu C. \& Dou C. 2014. Discussion on the wireless power transmission and new applications. Information Systems Engineering, (3): 94-94.

[9] Chaari, M. zied: Design and production of a system for wirelessly charging the batteries of a robot. 
[10] Chaari, M.Z., Al-maadeed, S.: Wireless Power Transmission for the Internet of Things (IoT). In: 2020 IEEE International Conference on Informatics, IoT, and Enabling Technologies (ICIoT). pp. 549554 IEEE, Doha, Qatar (2020). https://doi.org/10.1109/iciot48696. $\underline{2020.9089547}$

[11] Amici, T.T. et al.: Augmented Reality Applied to a Wireless Power Measurement System of an Industrial 4.0 Advanced Manufacturing Line. In: 2018 13th IEEE International Conference on Industry Applications (INDUSCON). pp. 14021406 IEEE, So Paulo, Brazil (2018). https://doi.org/10.1109/induscon.2018.8627301

[12] Grabia, M. et al.: Design of a DASH7 low power wireless sensor network for Industry 4.0 applications. In: 2017 IEEE International Conference on RFID Technology \& Application (RFID-TA). pp. 254259 IEEE, Warsaw (2017). https://doi.org/10.1109/rfid-ta.2017. $\underline{8098904}$

[13] Benzi, F. et al.: Wireless Power Sensors to Renovate Energy Metering in IIoT Converted Factories. In: 2019 II Workshop on Metrology for Industry 4.0 and IoT (MetroInd4.0\&IoT). pp. 414419 IEEE, Naples, Italy (2019). https://doi.org/10.1109/metroi4. $\underline{2019.8792844}$

[14] Wu, H. et al.: Wireless Powered Mobile Edge Computing for Industrial Internet of Things Systems. IEEE Access. 11 (2020). https://doi.org/10.1109/ACCESS.2020.2995649

[15] Xin Zan, Al-Thaddeus Avestruz, Wireless power transfer for implantable medical devices using piecewise resonance to achieve high peak-to-average power ratio, IEEE 18th Workshop on Control and Modeling for Power Electronics (COMPEL), USA, July 2017. https://doi.org/10.1109/compel.2017.8013365

[16] Chaari, M.Z., Al-Maadeed, S.A.: Spiral Antenna Mounted on the t-Shirt to Har- vested RF Energy. In: 2019 1st International Conference on Electrical, Control and Instrumentation Engineering (ICECIE). pp. 16 IEEE, Kuala Lumpur, Malaysia (2019). https://doi.org/ $\underline{10.1109 / i c e c i e 47765.2019 .8974756}$

[17] Ting, J.J.-L.: Proposal for verifying dipole properties of light- harvesting antennas. J. Photochem. Photobiol. B. 179, 134138 (2018). https://doi.org/10.1016/j.jphotobiol.2018.01. $\underline{011}$

[18] Antenna theory: analysis and design / Constantine A. Balanis, third edition, Hobo- ken, NJ: Wiley, 2005, ISBN 047166782X (hbk.)

[19] Hirose, K. et al.: A Loop-Line Antenna with Wideband Circular Polarization. In: 2018 IEEE International Symposium on Antennas and Propagation \& USNC/URSI National Radio Science Meeting. pp. 19331934 IEEE, Boston, MA (2018). https://doi.org/10.1109/ apusncursinrsm.2018.8608496

[20] Colaiuda, D. et al.: Rectifiers Design and Optimization for a Dual-Channel RF Energy Harvester. J. Low Power Electron. Appl. 10, 2, 11 (2020). https://doi.org/10.3390/jlpea $\underline{10020011}$

[21] Chaari, M.Z.: Transfert dnergie lectrique pour charger les batteries dun robot Transmission dnergie. Presses Acadmiques Francophones, Saarbrcken (2015).

[22] Park, Y. et al.: Rectenna Array Design for Receiving High Power in Beam Type Wireless Power Transmission. In: 2018 Asia-Pacific Microwave Conference (APMC). pp. 440442 IEEE, Kyoto (2018). https://doi.org/10.23919/apmc.2018.8617448

[23] Chalise, S. et al.: 45\% RF-to-DC Conversion Efficiency Wireless Power Transfer System Through Biological Tissues Using Complex Conjugate Impedance Matching Taking Ac- 
count of Tissues Properties. In: 2019 IEEE MTT-S International Microwave Symposium (IMS). pp. 14191422 IEEE, Boston, MA, USA (2019). https://doi.org/10.1109/mwsym. 2019.8700821

[24] Sedeek, A. et al.: Design of an efficient $2.45 \mathrm{GHz}$ RF rectifier for energy harvesting from low RF power density environment. In: 2020 International Conference on Innovative Trends in Communication and Computer Engineering (ITCE). pp. 268271 IEEE, Aswan, Egypt (2020). https://doi.org/10.1109/itce48509.2020.9047817

\section{$7 \quad$ Authors}

Mohamed Zied Chaari and Somaya Al-Maadeed are from Qatar University, Doha, Qatar. chaari zied@ieee.org

Article submitted 2021-04-21. Resubmitted 2021-05-21. Final acceptance 2021-05-30. Final version published as submitted by the authors. 